\title{
$\mathbf{R}$
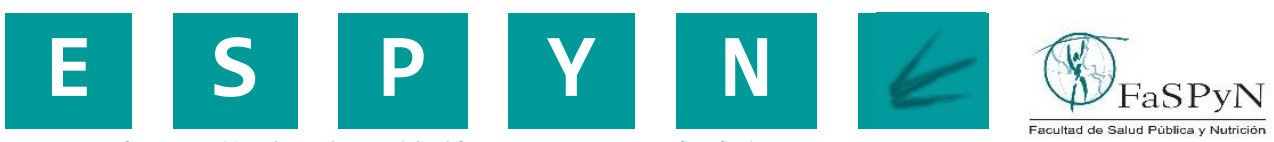

Revista Salud Pública y Nutrición

\section{FACTORES RELACIONADOS A LA PRESENCIA DEL SÍNDROME DE BURNOUT EN UNA MUESTRA DE ENFERMERAS DEL SECTOR SALUD, CIUDAD DE MÉXICO.}

\author{
FACTORS RELATED TO THE PRESENCE OF BURNOUT SYNDROME IN A SAMPLE OF NURSES IN THE \\ HEALTH SECTOR, MEXICO CITY.
}

Sánchez-Jiménez Bernarda ${ }^{1}$, Flores-Ramos Mónica ${ }^{2}$, Sámano Reyna ${ }^{1}$, Rodríguez-Ventura Ana Lilia ${ }^{1}$, Chinchilla Ochoa Daniela ${ }^{1}$.

1 Instituto Nacional de Perinatología "Isidro Espinosa de los Reyes", México. 2 Instituto Nacional de Psiquiatría Ramón de la Fuente Muñiz, México.

Citation: Sánchez-Jiménez B., Flores-Ramos M., Sámano R., RodríguezVentura A.L., Chinchilla Ochoa D. (2018) Factores relacionados a la presencia del síndrome de Burnout en una muestra de enfermeras del Sector Salud, Ciudad de México. Revista de Salud Pública y Nutrición, 17(3), 1-8.

Editor: Esteban G. Ramos Peña, Dr. CS., Universidad Autónoma de Nuevo León, Facultad de Salud Pública y Nutrición, Monterrey Nuevo León, México. Copyright: (C2018 Flores-Ramos M. et al. This is an open-access article distributed under the terms of Creative Commons Attribution License [CC BY 4.0], which permits unrestricted use, distribution, and reproduction in any medium, provided the original author and source are credited.

Competing interests: The authors have declared that no competing interests exist.

DOI: https://doi.org/10.29105/respyn17.3-1

Recibido: 21 de junio 2018;

Aceptado: 21 de septiembre 2018

Email: flores ramos@hotmail.com 


\title{
FACTORES RELACIONADOS A LA PRESENCIA DEL SÍNDROME DE BURNOUT EN UNA MUESTRA DE ENFERMERAS DEL SECTOR SALUD, CIUDAD DE MÉXICO.
}

\author{
Sánchez-Jiménez Bernarda ${ }^{1}$, Flores-Ramos Mónica ${ }^{2}$, Sámano Reyna ${ }^{1}$, Rodríguez-Ventura Ana Lilia ${ }^{1}$, \\ Chinchilla Ochoa Daniela ${ }^{1}$.
}

\author{
1 Instituto Nacional de Perinatología “Isidro Espinosa de los Reyes". 2 Instituto Nacional de Psiquiatría Ramón de la
} Fuente Muñiz.

\section{RESUMEN}

Introducción: Las enfermeras son un grupo profesional con alto riesgo de sufrir síndrome de Burnout. Algunos factores sociodemográficos y psicológicos se relacionan a dicho síndrome. Objetivo: evaluar la relación entre el estrés psicológico, los tres factores descritos para el síndrome de Burnout, y las características socio-demográficas y psicológicas en un grupo de enfermeras. Material y Métodos: Participaron 265 enfermeras de un centro especializado en salud reproductiva. Todas completaron las evaluaciones sobre características socio-demográficas y aspectos relacionados con sus condiciones de trabajo. Se aplicaron además el Inventario de Burnout de Maslach, el Inventario de Coopersmith, y el Cuestionario General de Salud. Resultados: Se observaron altos niveles de agotamiento emocional en $17.4 \%$ de la muestra. El $4.9 \%$ de las enfermeras mostraron bajos niveles de realización personal. La autoestima estuvo relacionada con el agotamiento emocional, la despersonalización y la realización personal. El agotamiento emocional se relacionó con la atención de pacientes seriamente enfermos, y con el trabajo en el horario matutino. Conclusiones: Los factores del síndrome de Burnout, particularmente el agotamiento emocional se relaciona con las condiciones de trabajo. Otros elementos que se relacionan con este síndrome son el estrés psicológico y la autoestima.

Palabras Clave: Burnout, enfermera, autoestima.

\section{ABSTRACT}

Introduction: Nurses are an important population in high risk to suffer Burnout. Some working factors, socio-demographic variables and psychological variables are related to the Burnout syndrome. Objective: To evaluate the relation between the three factors of Burnout and psychological distress with socio-demographic characteristics, working and psychological characteristics and of a sample of nurses. Methods: Participants were 265 nurses of a specialized institute in reproductive health. All of them completed the instruments about socio-demographic characteristics and specific aspects of working conditions. The Maslach Burnout Inventory, the Coopersmith Inventory, and the General Health Questionnaire were applied to the participants. Results High levels of emotional exhaustion were observed in $17.4 \%$ of the sample. The $4.9 \%$ of the nurses showed a low level of personal accomplishment. Self-esteem was related to emotional exhaustion, depersonalization, and personal accomplishment. Emotional exhaustion was related to seriously ill patient attention, and morning hours of work. Conclusions: Burnout syndrome factors, especially emotional exhaustion are related to working conditions. Psychological distress and self-esteem are also related to Burnout syndrome.

Key words: Burnout, nurses, self-esteem. 


\section{Introducción}

El síndrome de Burnout fue descrito como el resultado de una respuesta al estrés prolongado, originado por una serie de estresores crónicos emocionales e interpersonales (Maslach, Schaufeli, \& Leiter, 2001). Dicho síndrome consiste en la presencia de agotamiento emocional, despersonalización, y sensación de pobre realización personal ante la exposición a estrés crónico. Se ha considerado que el agotamiento emocional es el principal componente del síndrome de Burnout, y se caracteriza por la falta o carencia de energía y entusiasmo (Maslach et al., 2001); mientras que la despersonalización es una actitud distante y cínica hacia el trabajo; la realización personal disminuida se define como el sentimiento de no ser capaz de hacer el trabajo con eficacia. Con base en la descripción del síndrome de Burnout, se creó el inventario de evaluación de Maslach, que evalúa los tres factores de este síndrome: agotamiento emocional, despersonalización y realización personal (Maslach \& Jackson, 1981) y este instrumento ha sido utilizado para evaluar a poblaciones con diferentes profesiones. El personal de salud ha mostrado ser más vulnerable a sufrir síndrome de Burnout; particularmente en el caso de las enfermeras se ha documentado una alta prevalencia de este síndrome (Raftopoulos, Charalambous, \& Talias, 2012), lo cual probablemente se derive del contacto cercano con pacientes de difícil manejo, o del temor a cometer errores en el cuidado de la salud (Ferreira \& Lucca, 2015). De igual manera pueden ser factores moduladores del estrés la ambigüedad en el rol que se cumple, la carga de trabajo, la edad, la resiliencia, un estilo de afrontamiento activo y el apoyo social (Duquette, Kérowc, Sandhu, \& Beaudet, 1994). Por otro lado, es importante evaluar el papel de la satisfacción laboral (Kalliath \& Morris, 2002), de la motivación, el liderazgo, el empoderamiento y la confianza (Papathanasiou et al., 2014) como factores que pueden disminuir la presencia de Burnout en personal dedicado a la enfermería. Tanto la satisfacción personal como los niveles de Burnout pueden estar determinados por condiciones laborales como estresores en el sitio de trabajo (Lambert, Lambert, Petrini, Li, \& Zhang, 2007), como pueden ser el control de los insumos, el equipamiento inadecuado o pobre, las condiciones de seguridad inadecuadas en el sitio de trabajo, el manejo del personal y las dificultades para adherirse al presupuesto del hospital (Khamisa, Oldenburg,
Peltzer, \& Ilic, 2015). Sin embargo, no solo las condiciones laborales sino también otros factores personales pueden contribuir a desarrollar un síndrome de Burnout, de los cuales podemos mencionar el nivel educativo (Lu, While, \& Barriball, 2007) y factores psicológicos propios de la persona (Chakraborty, Chatterjee, \& Chaudhury, 2012). Algunos autores han sugerido que la autoestima también puede contribuir a la satisfacción laboral, pero no se ha corroborado una relación directa entre esta variable y el síndrome de Burnout (Chakraborty et al., 2012; Lou, Li, Yu, \& Chen, 2011).

El en caso del estrés psicológico se ha observado que tiene una relación directa con los niveles de agotamiento emocional y una relación inversa con la satisfacción personal. En una muestra de médicos dedicados a la medicina de urgencias se observó que el estrés psicológico fue altamente prevalente y que el Burnout correlacionó significativamente, de manera inversa, con la satisfacción laboral (Boudreaux, Mandry, \& Brantley, 1997). De manera similar, en una evaluación realizada a personal de enfermería que incluyó a 4,407 enfermeras de 8 hospitales generales de Japón, se observó que un alto porcentaje $(68 \%)$ sufrían de estrés psicológico de acuerdo al Cuestionario General de Salud (Suzuki et al., 2004). Una observación importante en este estudio fue la asociación entre una "pobre salud mental" y la presencia de errores médicos en los 12 meses previos. Concluyendo los autores que la importante labor de los profesionales de la salud, particularmente las enfermeras que se encuentran en contacto directo con sus pacientes, implica que deben encontrarse en un "buen estado mental". Por ello, es de suma importancia entender qué factores afectan la salud mental de este personal, para poder planear intervenciones de índole emocional a fin de fortalecer u optimizar su estado de salud, lo cual puede reducir el ausentismo laboral y permitir una mayor productividad; además, mejorarían la ejecución de sus funciones en su praxis diaria con sus pacientes y población en general.

En virtud de lo anterior, el objetivo del presente trabajo fue explorar los niveles de Burnout y estrés psicológico en un grupo de enfermeras dedicadas a la salud reproductiva y su relación con variables sociodemográficas, auto-estima y variables relacionadas con las condiciones laborales. Nuestra 
hipótesis es que los niveles de Burnout y el estrés psicológico se encontrarán relacionados con condiciones laborales negativas y características sociodemográficas desfavorables de las participantes.

\section{Material y Métodos}

Diseño del estudio

Estudio transversal y analítico, realizado de 2013 y 2016 en un grupo de enfermeras de una institución dedicada particularmente a la atención de mujeres con embarazo de alto riesgo, pero cuenta con muchos otros servicios de atención médica para la mujer y el neonato. La muestra fue consecutiva e intencional en enfermeras que tuvieran un contrato de base, de todos los turnos, servicios y categorías existentes en el Instituto. Fueron convocadas mediante invitación personal por la investigadora principal y solamente aquéllas(os) que de manera voluntaria aceptaron participar fueron incluidos en el estudio, firmando carta de consentimiento. La muestra final quedó constituida por 265 participantes de un universo de 478 enfermeras(os). El total de participantes que constituyeron la muestra final fueron quienes tenían disponibilidad de tiempo y acudieron a la evaluación. Se excluyeron a los enfermeros hombres en virtud de que constituían menos del $1 \%$ del total de los entrevistados; así mismo se excluyeron embarazadas y participantes que dejaran sus cuestionarios incompletos $\mathrm{y} / \mathrm{o}$ con datos inconsistentes. Este proyecto fue aprobado por los Comités de Ética, de Investigación y Bioseguridad de nuestra Institución.

\section{Evaluaciones}

Características socio-demográficas.

Se registró su estado civil, formación académica y religión en formato elaborado para tal fin. El nivel socio-económico fue medido con un cuestionario especial desarrollado por la Asociación Mexicana de Investigación de Mercado y Opinión Pública. Este cuestionario consta de 10 ítems que al ser calificados se agrupan en 6 niveles socioeconómicos: muy bajo, bajo, medio-bajo, medio-medio, medio-alto, alto y muy alto (López Romo, 2011).

Condiciones laborales.

Las variables evaluadas en relación a las condiciones laborales fueron: años de servicio en la institución, años de servicio en otra institución dado el caso de haber laborado previamente; turno de trabajo que se clasificó como matutino, vespertino y nocturno. Tipo de pacientes atendidos de acuerdo con la enfermedad, gravedad de los pacientes y tipo de servicio (incluyendo asistencia directa a pacientes o trabajo predominantemente administrativo). Además, se registró si las enfermeras estaban trabajando en otra institución académica o de salud además de la nuestra.

\section{Cuestionarios.}

Los 30 ítems del Cuestionario General de Salud (CGS-30) fueron usados para evaluar el estrés psicológico. Este fue originalmente desarrollado por Goldberg y ha sido ampliamente utilizado en diferentes estudios como una herramienta para determinar si un individuo se encuentra en riesgo de desarrollar trastornos psiquiátricos (Golderberg \& Williams, 1988); versiones posteriores de este instrumento se han desarrollado con cambios en el número de ítems, estas versiones muestran propiedades clinimétricas adecuadas para usarse en escenarios médicos. Una versión mexicana de la escala fue validada desde 1983 y ha sido utilizada en la población mexicana (Golderberg \& Williams, 1988).

Para evaluar la auto-estima se utilizó el inventario de Coopersmith (Mikesell, Calhoun, \& Lottman, 1970) que es un instrumento de 25 reactivos que describen actitudes hacia uno mismo y hacia los demás, así como intereses personales. Una versión mexicana de este inventario fue validada para ser usada en adultos (Cantú, Asunción, Verduzco, Acevedo, \& Cortés, 1993), y mostró un coeficiente de confiabilidad de 0.81 usando Alfa de Cronbach.

El Inventario de Maslach de Burnout (MBI, por sus siglas en inglés) se utiliza para describir el estrés laboral en profesionales que se encuentran en contacto directo con la gente. Comprende 22 ítems que evalúan las tres dimensiones básicas: agotamiento emocional, despersonalización, y satisfacción personal. Las puntuaciones iguales o mayores a 27 indican un agotamiento emocional elevado; puntuaciones de 17 a 26 indican agotamiento emocional moderado y las puntuaciones de 16 o menos indican un bajo grado de agotamiento emocional. Con respecto a la despersonalización las puntuaciones de 13 o más indican un estado severo de despersonalización, mientras que puntuaciones de 7 a 12 se consideran moderadas y puntuaciones de 6 o menos indican bajo grado de despersonalización 
(Cooper, 1998). La puntuación para satisfacción personal se invierte, siendo los puntajes de 0 a 31 indicativos de severidad, mientras que puntuaciones de 32 a 38 son moderados y puntuaciones superiores a 39 indican un bajo grado de satisfacción personal (a mayor puntuación menor afectación de la persona y mayor realización personal). La versión en español del inventario de Maslach fue validada y demostró tener una validez interna elevada y una adecuada confiabilidad con una alfa de Cronbach de 0.9 (Moreno-Jiménez, Bustos, Matallana, \& Miralles, 1997).

Análisis estadístico

Se reporta el análisis descriptivo de las variables. Se llevó a cabo también un análisis de correlación entre los factores de Burnout y las variables sociodemográficas y de las condiciones laborales, utilizando la prueba de correlación de Spearman. Un análisis de la varianza o una prueba de Kruskal Wallis, según correspondía, se utilizaron para comparar las puntuaciones de los tres factores o dimensiones de Burnout dependiendo del tipo de paciente atendido, y el turno de trabajo. Los niveles de significancia estadística fueron establecidos en 0.05. Utilizamos el programa estadístico SPSS versión 12.0 para llevar a cabo el análisis de datos.

\section{Resultados}

De un total de 478 enfermera(o)s convocados, 282 respondieron los cuestionarios; es decir obtuvimos una tasa de respuesta de 58.99\%. De éstos se excluyeron los datos de 2 hombres, dos enfermeras embarazadas y 13 cuestionarios incompletos o con datos inconsistentes. La muestra final fue de 265 participantes que completaron los cuestionarios correctamente. La media de edad de las enfermeras fue de 41 años, las características socio-demográficas de las participantes se muestran en la tabla 1. Con respecto a las condiciones laborales observamos que el tiempo promedio de laborar en esta institución fue de 18 años (de=8.5); 145(54.7\%) de las participantes se encontraban laborando en el turno matutino, $45(17 \%)$ en el turno vespertino, y $75(28.3 \%)$ en el nocturno. De todas las participantes el $7.9 \%$ se encontraban además laborando en algún otro centro académico o de atención a la salud como hospitales o consultorios médicos. El $63.8 \%$ de las enfermeras evaluadas se encontraban en contacto directo con pacientes gravemente enfermos, $18.5 \%$ atendían pacientes hospitalizados con entidades medicas no graves, $8.7 \%$ se encontraban asignadas a los servicios de consulta externa, y $9.1 \%$ tenían labores predominantemente administrativas y poco contacto con pacientes.

Tabla 1. Características socio-demográficas de las participantes.

\begin{tabular}{|c|c|c|}
\hline & $\mathrm{N}$ & $\%$ \\
\hline \multicolumn{3}{|l|}{ Estado civil } \\
\hline Casada o unión libre & 166 & 63 \\
\hline Soltera o viuda & 99 & 37 \\
\hline \multicolumn{3}{|l|}{ Turno de trabajo } \\
\hline Matutino & 145 & 54.7 \\
\hline Vespertino & 45 & 17 \\
\hline Nocturno & 75 & 28.3 \\
\hline \multicolumn{3}{|l|}{ Años de servicio } \\
\hline ene-14 & 76 & 28.7 \\
\hline $15-29$ & 168 & 63.4 \\
\hline$\geq 30$ & 21 & 7.9 \\
\hline \multicolumn{3}{|l|}{ Nivel socio-económico } \\
\hline Bajo y Medio-bajo & 17 & 6.4 \\
\hline Medio-medio & 73 & 27.6 \\
\hline Medio-alto & 119 & 44.9 \\
\hline Alto & 56 & 21.1 \\
\hline \multicolumn{3}{|l|}{ Religión } \\
\hline Católica & 240 & 91 \\
\hline Otra & 25 & 9 \\
\hline
\end{tabular}

Las puntuaciones promedio de agotamiento emocional, despersonalización y realización personal fueron $17.49,2.7$ y 43.64 respectivamente. Cuando categorizamos estas variables encontramos que $17.4 \%$ de las enfermeras tenían un alto nivel de agotamiento emocional, $1.1 \%$ presentaron un alto grado de despersonalización, y $4.9 \%$ tenían un bajo nivel de realización personal. Niveles bajos de autoestima fueron observados en un total de 71 enfermeras (26.8\%), y altos niveles de estrés psicológico fueron observados en 27 enfermeras $(10.2 \%)$.

El agotamiento emocional no se correlacionó con el turno de trabajo, el nivel educativo, los años de servicio en esta institución, ni el hecho de estar laborando en otro centro de atención a la salud. Nosotros observamos que el agotamiento emocional correlacionó de manera significativa pero inversa 
con las puntuaciones de auto-estima (rho de Spearman $=-0.264, \mathrm{p}<0.05)$, indicando que a mayor autoestima menor agotamiento emocional. Cuando comparamos los grupos de acuerdo al tipo de pacientes atendidos observamos que las puntuaciones de agotamiento emocional eran mayores en enfermeras que atendían a pacientes seriamente enfermos en comparación a enfermeras asignadas a la consulta externa (17vs11; Kruskal Wallis, $\mathrm{p}=0.020$ ); así mismo observamos que las enfermeras trabajando en un horario matutino tenían puntuaciones mayores de agotamiento emocional en comparación a las enfermeras que trabajaban por la tarde sin ser esta una diferencia estadísticamente significativa (17 vs 13; Kruskal Wallis, $\mathrm{p}=0.06$ ) (tabla 2).

La despersonalización no mostró correlación con las variables socio-demográficas, y al igual que el agotamiento emocional, mostró una relación inversa con la auto-estima (rho de Spearman $=0.196, \mathrm{p}<$ 0.001). Los años de servicio se correlacionaron de manera inversa a la despersonalización (rho de Spearman $=-0.205, \mathrm{p}<0.001)$.

En el caso de la realización personal, mostró una correlación directa con la auto-estima (rho de Spearman $=0.298, \mathrm{p}<0.001)$. Aun cuando no observamos diferencias en el promedio de puntuaciones de realización personal entre mujeres con o sin pareja, observamos que había una proporción mayor de mujeres solteras en el grupo de realización personal moderada o alta. Es de hacerse notar que la realización personal se correlacionó con el nivel socio-económico; los niveles socioeconómicos medio-alto, alto y muy alto mostraron puntuaciones significativamente mayores en autoestima en comparación a los otros niveles socioeconómicos. Igualmente, los niveles socioeconómicos medio-alto y alto presentaron puntuaciones significativamente mayores en realización personal en comparación a los niveles medio bajo y bajo (ANOVA, Tukey $\mathrm{p}=0.030,0.034$ ). La realización personal no mostró correlación con las variables laborales evaluadas como años de servicio, horarios de trabajo y tipo de pacientes atendidos.

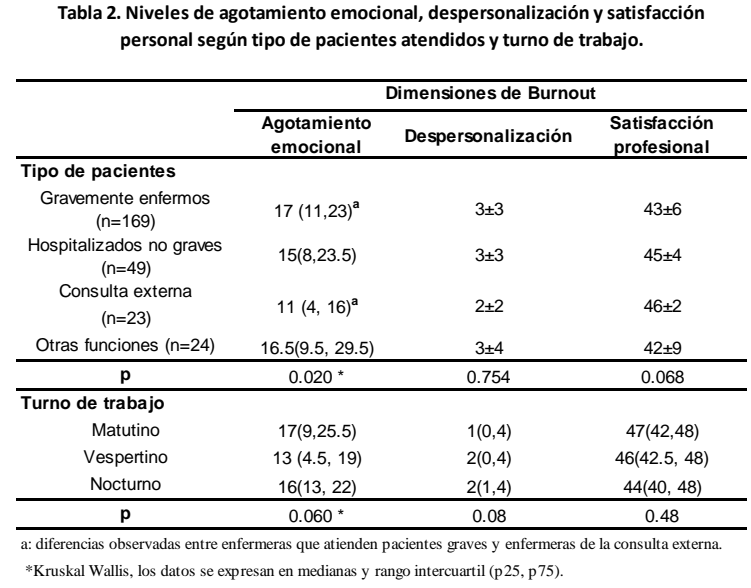

El estrés psicológico medido de acuerdo al CGS-30 estuvo directamente relacionado con el agotamiento emocional y la despersonalización, e inversamente relacionado con la auto-estima y las puntuaciones de realización personal.

\section{Discusión}

Un elevado nivel de Burnout fue observado en esta muestra de enfermeras, acorde con lo reportado por otros autores. Particularmente, nosotros observamos una proporción elevada de enfermeras con altos niveles de agotamiento personal y esto se relacionó con el tipo de pacientes atendidos. En nuestra muestra, la atención de pacientes gravemente enfermos se relacionó con altos niveles de agotamiento emocional. Diversos estudios han reportado proporciones similares de enfermeras con síndrome de Burnout (Poncet et al., 2007), e incluso mayor porcentaje llegando hasta dos tercios del personal de enfermería dedicado al cuidado de pacientes en estado crítico con síndrome de Burnout (Cicchitti, Cannizzaro, Rosi, Maccaroni, \& Menditto, 2014). Las comparaciones entre personal de salud dependiendo del tipo de servicio que presten, han demostrado que el personal que labora en unidades de emergencia o unidades de cuidado a pacientes en estado crítico son más propensos a sufrir de Burnout (Ahmadi, Azizkhani, \& Basravi, 2014). En el caso de las participantes de este estudio, un alto porcentaje atiende pacientes con un estado delicado de salud, debido a que es el tipo de pacientes que habitualmente se atienden en este hospital, esto podría ser un motivo por el cual existen altos niveles de Burnout. 
Contrariamente a lo que esperábamos, las enfermeras que trabajaban en el turno matutino tenían altas puntuaciones de agotamiento emocional. Nosotros consideramos que independientemente del turno de trabajo, el número de horas trabajadas por semana debería ser evaluado, dado que sabemos que en nuestro sistema de salud los horarios vespertinos y nocturnos implican menos horas de trabajo a la semana. En el caso de nuestro hospital, las enfermeras que laboran en el horario matutino permanecen en el sitio de trabajo aproximadamente 40 horas por semana, mientras que aquellas que trabajan en el horario vespertino tienen asignadas entre 30 a 35 horas semanales. De manera similar a lo que nosotros observamos, un estudio brasileño encontró que trabajar en el horario matutino con un promedio de 36 horas semanales fue un factor relacionado a síndrome de Burnout (Ribeiro et al., 2014). En el mismo contexto, un estudio reporta que la demanda de trabajo está asociada con el promedio de tiempo que las enfermeras se encuentran en el hospital (Cho, Park, Jeon, Chang, \& Hong, 2014), por lo cual hubiera sido importante evaluar cuál es la percepción de la muestra que evaluamos en cuanto a la demanda de trabajo dependiendo del turno laborado.

Existen escasos datos acerca de la relación entre auto-estima y Burnout, en una exploración piloto de la relación entre la auto-estima y la satisfacción profesional, se detectó una correlación positiva entre estas variables, con un efecto mediador sobre el síndrome de Burnout (Karanikola, Papathanassoglou, Giannakopoulou, \& Koutroubas, 2007). En el mismo sentido, una encuesta de 253 enfermeras reportó una correlación positiva entre auto-estima y satisfacción laboral (Moore, Lindquist, \& Katz, 1997). Acorde con estos resultados, nuestra muestra la autoestima estuvo relacionada con los factores del síndrome de Burnout, las enfermeras con altos niveles de realización personal presentaban mayor autoestima, y las enfermeras con altos niveles de agotamiento emocional y despersonalización presentaban bajas puntuaciones en la medición de autoestima. Debido al diseño transversal de nuestro trabajo no podemos asumir una relación causal entre autoestima y los tres factores del Burnout, ni identificar si las puntuaciones en los factores de Burnout son causa o consecuencia del nivel de autoestima; sin embargo, es importante señalar existe una relación entre Burnout y autoestima. Es necesario considerar que estar sometido crónicamente a estrés podría impactar en la autoestima de los trabajadores. Estudios posteriores de seguimiento, podrían clarificar en qué sentido se presenta esta relación y de esa manera poder proponer medidas para incidir en mejorar el bienestar del personal de enfermería.

Por último, nosotros observamos que la realización personal no se relaciona con las condiciones laborales. En este sentido, la variable que mostró mayor relación con la realización personal fue el nivel socio-económico. Estudios previos han sugerido que el desbalance entre el esfuerzo y la recompensa está asociado con el síndrome de Burnout. Se sabe que la percepción económica es una recompensa importante en el área laboral, lo que podría explicar los hallazgos encontrados. Un estudio realizado en siete países europeos encontró que una proporción desbalanceada entre esfuerzo y recompensa se relacionó con el síndrome de Burnout y con la intención de abandonar la profesión de enfermería (Hasselhorn, Tackenberg, \& Peter, 2004). En otro estudio, llevado a cabo con enfermeras iraníes, el factor que afectó de manera más importante para sufrir Burnout fue la desproporción entre tiempo de trabajo e ingreso (Moghaddasi, Mehralian, Aslani, Masoodi, \& Amiri, 2013).

La seguridad de los pacientes en servicios médicos, se encuentra comprometida cuando el personal de salud se encuentra con altos niveles de Burnout, como lo describen Welp y colaboradores (Welp, Meier, \& Manser, 2015). En el estudio de los autores mencionados en el que participaron 1425 sujetos (entre médicos y enfermeras), se observó que los niveles elevados de agotamiento emocional eran predictores de mortalidad en los pacientes; en las unidades en que el personal tenía altos niveles de agotamiento emocional existían mayores tasas estandarizadas de mortalidad. De ahí que se considera que la importante labor del personal de salud, requiere que exista una óptima salud mental en ellos y, por tanto, es importante entender qué factores afectan la salud mental de dicho personal, para poder establecer estrategias que den soporte emocional al personal de enfermería. Además de evaluar las condiciones laborales, debemos considerar los satisfactores que pueden modular el estrés, como el reconocimiento a la labor de enfermería e incentivos 
para personal que atienda pacientes críticamente enfermos.

\section{Conclusiones}

Los factores que contribuyeron a la presencia de Burnout en esta muestra de enfermeras fueron el trabajo en el turno matutino y la atención de pacientes gravemente enfermos. La autoestima es otro factor que mostró relación con el Burnout, sin embargo, es importante realizar más estudios que puedan determinar cómo se comporta dicha relación. Finalmente, un bajo nivel socioeconómico se relacionó con insatisfacción personal de las participantes.

\section{Bibliografía}

Ahmadi, O., Azizkhani, R., y Basravi, M. (2014). Correlation between workplace and occupational burnout syndrome in nurses. Advanced biomedical research, 3.

Boudreaux, E., Mandry, C., y Brantley, P. J. (1997). Stress, job satisfaction, coping, and psychological distress among emergency medical technicians. Prehospital and Disaster Medicine, 12(4), 9-16.

Cantú, L., Asunción, M., Verduzco, M. A., Acevedo, M., \& Cortés, J. (1993). Validez y confiabilidad del inventario de autoestima de Cooper Smith para adultos, en población mexicana. Revista latinoamericana de psicología, 25(2).

Cicchitti, C., Cannizzaro, G., Rosi, F., Maccaroni, R., \& Menditto, V. (2014). Burnout syndrome in pre-hospital and hospital emergency. Cognitive study in two cohorts of nurses. Recenti progressi in medicina, 105(7-8), 275-280.

Cooper, C. L. (1998). Theories of organizational stress: OUP Oxford.

Chakraborty, R., Chatterjee, A., y Chaudhury, S. (2012). Internal predictors of burnout in psychiatric nurses: An Indian study. Industrial psychiatry journal, 21(2), 119.

Cho, S. H., Park, M., Jeon, S. H., Chang, H. E., y Hong, H. J. (2014). Average hospital length of stay, nurses' work demands, and their health and job outcomes. Journal of Nursing Scholarship, 46(3), 199-206.

Duquette, A., Kérowc, S., Sandhu, B. K., y Beaudet, L. (1994). Factors related to nursing burnout a review of empirical knowledge. Issues in Mental Health Nursing, 15(4), 337-358.
Ferreira, N. d. N., y Lucca, S. R. d. (2015). Burnout syndrome in nursing assistants of a public hospital in the state of São Paulo. Revista Brasileira de Epidemiologia, 18(1), 68-79.

Golderberg, D., y Williams, P. (1988). A user's guide to the General Health questionnaire. Windsor, UK: NFERNelson.

Hasselhorn, H.-M., Tackenberg, P., y Peter, R. (2004). Effort-reward imbalance among nurses in stable countries and in countries in transition. International journal of occupational and environmental health, 10(4), 401-408.

Kalliath, T., y Morris, R. (2002). Job satisfaction among nurses: a predictor of burnout levels. Journal of nursing administration, 32(12), 648-654.

Karanikola, M. N., Papathanassoglou, E. D., Giannakopoulou, M., y Koutroubas, A. (2007). Pilot exploration of the association between self-esteem and professional satisfaction in Hellenic Hospital nurses. Journal of Nursing Management, 15(1), 78-90.

Khamisa, N., Oldenburg, B., Peltzer, K., y Ilic, D. (2015). Work related stress, burnout, job satisfaction and general health of nurses. Int J Environ Res Public Health, 12(1), 652-666.

Lambert, V., Lambert, C., Petrini, M., Li, X., y Zhang, Y. (2007). Predictors of physical and mental health in hospital nurses within the People's Republic of China. International nursing review, 54(1), 85-91.

López Romo, H. (2011). Asociación Mexicana de Agencias de Investigación de Mercado y Opinión Pública (AMAI) Niveles Socioeconómicos (NSE) 8X7: PowerPoint.

Lou, J. H., Li, R. H., Yu, H. Y., y Chen, S. H. (2011). Relationships among self-esteem, job adjustment and service attitude amongst male nurses: A structural equation model. Journal of clinical nursing, 20(5-6), 864-872.

Lu, H., While, A. E., y Barriball, K. L. (2007). Job satisfaction and its related factors: a questionnaire survey of hospital nurses in Mainland China. International journal of nursing studies, 44(4), 574588.

Maslach, C., y Jackson, S. E. (1981). The measurement of experienced burnout. Journal of organizational behavior, 2(2), 99-113. 
Maslach, C., Schaufeli, W. B., \& Leiter, M. P. (2001). Job burnout. Annual review of psychology, 52(1), 397-422.

Medina-Mora, M., Padilla, G., Campillo-Serrano, C., Mas, C., Ezban, M., Caraveo, J., y Corona, J. (1983). The factor structure of the GHQ: a scaled version for a hopital's general practice service in Mexico. Psychological medicine, 13(2), 355-361.

Mikesell, R. H., Calhoun, L. G., \& Lottman, T. J. (1970). Instructional set and the Coopersmith Self-Esteem Inventory. Psychological reports, 26(1), 317-318.

Moghaddasi, J., Mehralian, H., Aslani, Y., Masoodi, R., y Amiri, M. (2013). Burnout among nurses working in medical and educational centers in Shahrekord, Iran. Iranian journal of nursing and midwifery research, 18(4), 294.

Moore, S., Lindquist, S., y Katz, B. (1997). Home health nurses: Stress, self-esteem, social intimacy, and job satisfaction. Home Care Provider, 2(3), 135-139.

Moreno-Jiménez, B., Bustos, R., Matallana, A., y Miralles, T. (1997). La evaluacion del burnout: Problemas y alternativas. Revista de Psicología del Trabajo y de las Organizaciones, 13 (2), 185, 207.

Papathanasiou, I. V., Fradelos, E. C., Kleisiaris, C. F., Tsaras, K., Kalota, M. A., y Kourkouta, L. (2014). Motivation, leadership, empowerment and confidence: Their relation with nurses' burnout. Materia sociomedica, 26(6), 405.

Poncet, M. C., Toullic, P., Papazian, L., Kentish-Barnes, N., Timsit, J.-F., Pochard, F., . . Azoulay, E. (2007). Burnout syndrome in critical care nursing staff. American journal of respiratory and critical care medicine, 175(7), 698-704.

Raftopoulos, V., Charalambous, A., \& Talias, M. (2012). The factors associated with the burnout syndrome and fatigue in Cypriot nurses: a census report. BMC Public Health, 12(1), 457.

Ribeiro, V. F., Ferreira Filho, C., Valenti, V. E., Ferreira, M., de Abreu, L. C., de Carvalho, T. D., . . . Leão, E. R. (2014). Prevalence of burnout syndrome in clinical nurses at a hospital of excellence. International archives of medicine, 7(1), 22.

Suzuki, K., Ohida, T., Kaneita, Y., Yokoyama, E., Miyake, T., Harano, S., . . . Tsutsui, T. (2004). Mental health status, shift work, and occupational accidents among hospital nurses in Japan. Journal of occupational health, 46(6), 448-454.
Welp, A., Meier, L. L., y Manser, T. (2015). Emotional exhaustion and workload predict clinician-rated and objective patient safety. Frontiers in psychology, 5, 1573. 